\title{
Improving Clinical Results in Veterinary Osseous Grafting
}

\author{
${ }^{1}$ Rocco E Mele, ${ }^{2}$ Gregori M Kurtzman, ${ }^{3}$ Lanka Mahesh
}

\begin{abstract}
Veterinary dentistry and oral surgery is beginning to incorporate osseous graft material and techniques into their everyday dental clinical practices. Autogenous, allogenic xenografts and synthetics are all part of a new armamentarium to improve clinical results and achieve osseous reconstruction either to preserve anatomical ridge contours or to support implants placed to replace teeth extracted or previously missing.

Clinicians and researchers have been exploring the use of calcium sulfate (CS) for over 100 years in orthopedic, spinal arthrodesis, and maxillofacial surgery. CS is an inexpensive, easy-to-use material that offers many advantages as a predictable and significant bone regeneration substrate.
\end{abstract}

Keywords: Bond apatite, Calcium sulphate, Immediate implant, Osseous graft, Ridge augmentation, Socket preservation.

How to cite this article: Mele RE, Kurtzman GM, Mahesh L. Improving Clinical Results in Veterinary Osseous Grafting. Int J Oral Implantol Clin Res 2017;8(2 \&3):65-71.

\section{Source of support: Nil}

Conflict of interest: None

\section{INTRODUCTION}

Morbidity, time, and expense with autografts have generated an abundance of alternative materials. Alternatives range from the simple, such as CS and calcium phosphate to the complex that contain allografts, bone morphogenetic proteins, and other agents. ${ }^{1}$

Clinicians and researchers have been exploring the use of CS for over 100 years in orthopedic, spinal arthrodesis, ${ }^{2}$ and maxillofacial surgery. ${ }^{3}$ Calcium sulfate is a simple inexpensive material that offers many advantages as a predictable and significant bone regeneration substrate. Calcium sulfate was first used as a filler for bone defects by Dreesman. ${ }^{4}$ Studies undertaken in the 1950s demonstrated that CS is an effective resorbable material for filling bone defects and retaining bone grafts. It was reported that it was rapidly resorbed, caused no inflammation, minimal

\footnotetext{
${ }^{1-3}$ Private Practitioner

${ }^{1}$ Veterinary Medicine, Tuson, Arizona, USA

${ }^{2}$ Silver Spring, Maryland, USA

${ }^{3}$ Department of Implantology, The Specialist Clinic, New Delhi India

Corresponding Author: Gregori M Kurtzman, Private Practitioner, Silver Spring, Maryland, USA, Phone: +3015983500, e-mail: drimplants@aol.com
}

foreign body response, and resulted in normal regenerated bone, with no measurable rise in serum calcium levels. ${ }^{5-8}$

Calcium sulfate has proven to be very versatile in bone repair and can be used alone as a defect filler or as a barrier over other graft material to prevent soft tissue ingrowth into the underlying graft material. The ability of CS to be easily absorbable makes it a good delivery vehicle for biomaterials, such as growth factors, osteogenic factors, and antibiotics. ${ }^{9}$ Currently, it is utilized to regenerate empty extraction sockets and to treat dehiscences and fenestrations. Calcium sulfate is also being utilized in oral surgery techniques, such as filling cysts, defect repair after removal of impacted teeth, periodontal defects, and as barrier membranes. ${ }^{10}$

\section{BOND APATITE ${ }^{\circledR}$ GRAFT MATE RIAL}

Bond apatite ${ }^{\circledR}$ (BA) is a composite graft that combines $2 / 3$ biphasic CS and 1/3 hydroxyapatite (HA) granules of different sizes and shapes. No additives, polymers or other chemicals are contained in the mix, so there is no alteration to the chemical structure of the CS. The ratio (2:1) of biphasic CS and HA in a specific particle size distribution takes advantage of each components properties. The CS component acts as a short-range space maintainer scaffold which completely degrades in relation to the bone formation rate in 4 to 10 weeks. However, the HA acts as a long-term space maintainer designed to slow down the overall resorption of the graft. The bioactivity and the graft transformation into vital bone are due to the biphasic CS component of the graft. The overall structure of BA has microporous (1-10 $\mu \mathrm{m})$ and macroporous $(50-500 \mu \mathrm{m})$ structure. The initial surface porosity is about $40 \%$, but as the CS completely degrades over time, it creates more space for the new bone to be formed, as the graft is converted to native bone. The graft porosity and its hygroscopic ability will allow the patient's blood into the graft immediately following placement.

The product is supplied in an all-in-one dual-chamber prefilled syringe containing the granulated powder in one chamber and physiological saline in the other chamber. Mixing the powder component with the liquid in the driver results in a viscous material that is suitable for injection into the graft site. Setting time is approximately 3 to 5 minutes. The material once mixed and injected into the site is moldable and hardens instantly in the presence of blood or saliva. It is recommended not to over-compress 
the material into the site as cement expansion may lead to patient discomfort. Following placement of the mixed material, a piece of dry gauze is placed over the material at the treatment site and pressed firmly for 3 seconds to wick any residual liquid and to compact the bone cement. The flap is then closed with sutures.

Due to the grafts' properties, use of a membrane is not required as long as primary closure can be achieved. Leaving the material exposed will result in material volume loss, decreasing the clinical results desired. But a gap of 1 to $3 \mathrm{~mm}$ is not an issue, as soft tissue will migrate rapidly over it and the gap will close in a few days. Use of a membrane is recommended in large defects in which soft tissue stabilization cannot be ensured or in socket preservation procedure when a flap was not reflected and the material is completely exposed to the oral environment. In those cases, a protective barrier is required over the graft to prevent volume loss.

Bond apatite ${ }^{\circledR}$ may be used in a wide variety of osseous defects, including medium- and large-sized defects, such as dehiscence, fenestration cases, periodontal bone defects, extraction socket augmentation, filling of bony defects, preimplant placement, or simultaneously with implant placement, filling a void postcyst removal, and ridge augmentations.

The 3-D Bond ${ }^{\mathrm{TM}}$ graft material is a graft binder cement made of pure biphasic CS. It is available in a syringe and following mixing with a liquid is directly injected into the site or can be mixed with other graft materials as a binder or as a barrier membrane over other materials. This graft was developed for smaller applications (socket augmentation) that has some bony wall support.
Radiographically, the appearance is completely different compared with other graft materials which appear radiopaque due to their constant presence in the grafted site. Initially, BA and 3-D bond appear radiopaque; gradually, a radiolucent appearance takes place, reflecting the graft transformation into newly formed osteoid before its calcification and integration with the surrounding bone become evident. After 4 to 8 weeks, the majority of the grafted site will appear radiolucent while spots remain, reflecting the presence of the HA particles. Within 12 to 16 weeks, radiopacity comparable to adjacent native bone takes place with the appearance of a natural trabecular form. New osteoid has already calcified.

\section{CLINICAL CASE SERIES}

\section{Case 1}

A 7-year-old F/S Basset hound presented with a left maxillary PM4 complicated fracture. A full thickness mucoperiosteal flap was elevated. The fractured tooth was sectioned and extracted. Following site debridement, a bone cement, BA (Augma Biomaterials; Monroe Township, NJ), was prepared in its premixed syringe and delivered to the extraction site. Primary closure was achieved and sutures were placed.

\section{RESULTS}

At 8 weeks postsurgery, the tissue had completely healed with maintenance of the alveolar ridge. The BA has completely filled the empty sockets and reestablished the alveolar crest contours (Figs 1 to 3 ).
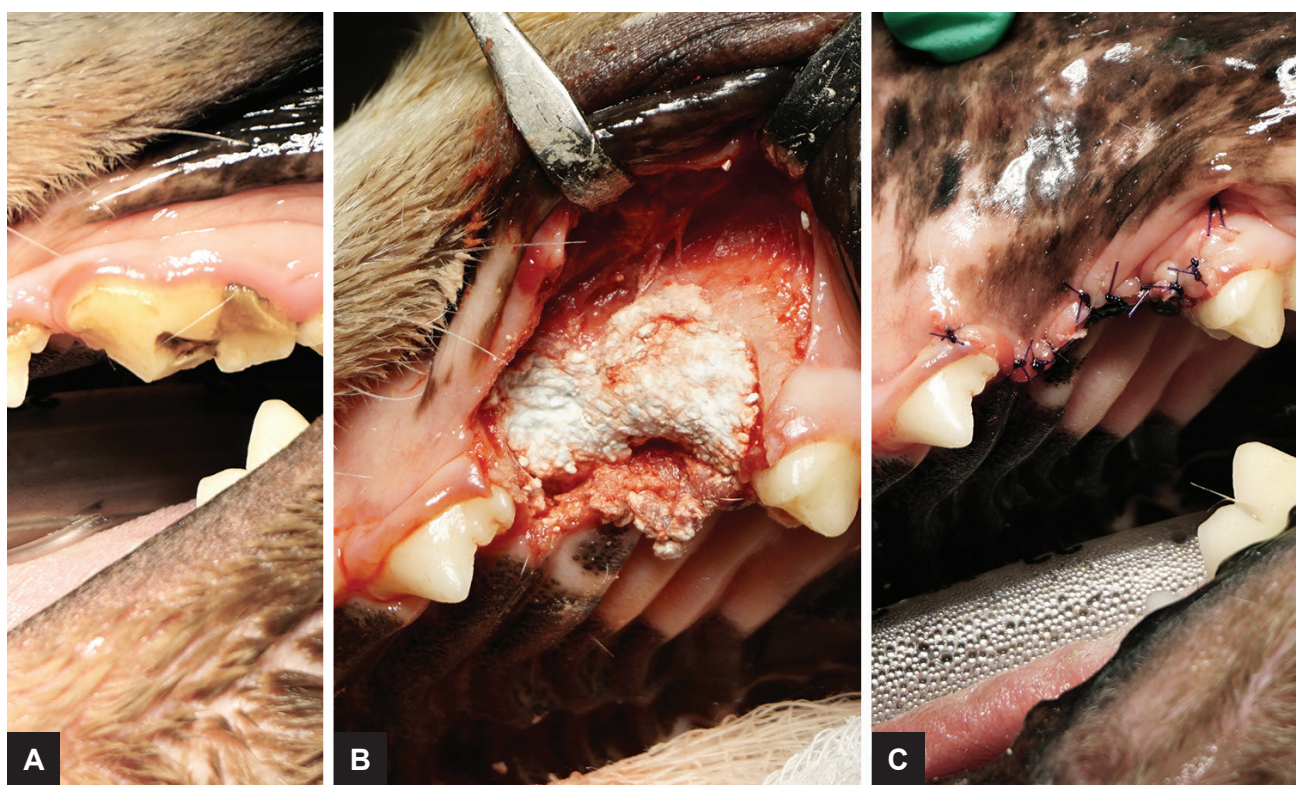

Figs 1A to C: (A) Complicated fracture left maxillary PM4; $(B)$ Molded BA in the prepared site to achieve normal anatomical contours to the ridge; (C) Primary closure and placement of sutures 

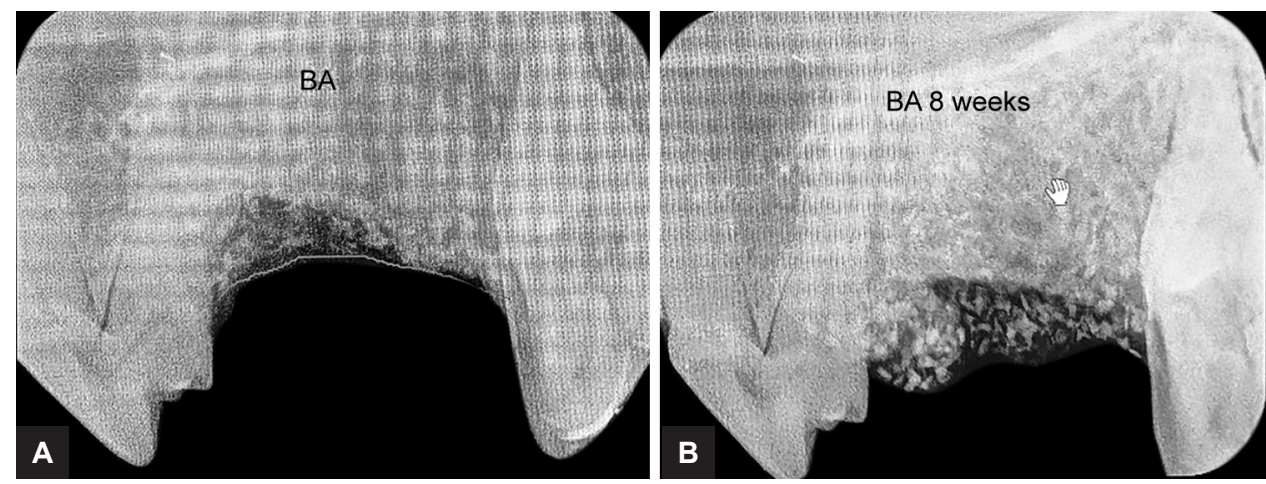

Figs 2A and B: (A) Radiograph of BA placed to rebuild the maxillary defect at the time of surgery; (B) 8-week radiolucent appearance, reflecting the graft transformation into newly formed osteoid before its calcification

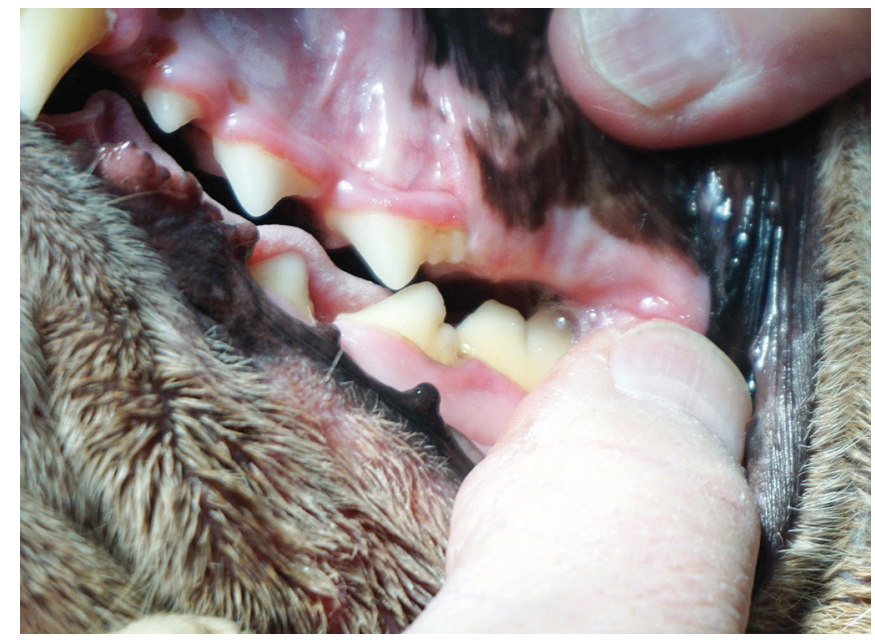

Fig. 3: Completely healed thick keratinized gingiva at 8 weeks postgrafting and maintenance of the ridge contour

\section{Case 2}

A 6-year-old $\mathrm{M} / \mathrm{N}$ feline (DLH) presented with a nonrestorable maxillary canine (\#204) fracture that was extracted and prepped for an immediate implant placement. Site was prepared following extraction with the Densah drill (Versah LLC, Jackson, MI) compacting drilling bone protocol. Following implant placement, a gap was noted between the implant and prepared site on the mesial and distal. Bond Apatite was utilized to fill the voids and to add to the crest to minimize resorption during the healing phase. Primary closure was achieved and the site closed with sutures.

\section{RESULTS}

The 6-month reentry to expose the implant to initiate the restorative phase confirmed soft and hard tissue health absent of any inflammatory reaction. The implant site demonstrated implant stability and ridge maintenance. No evidence of any graft particulate was noted, indicating complete conversion of graft to native bone. Radiograph confirmed complete osseointegration (Figs 4 to 6).
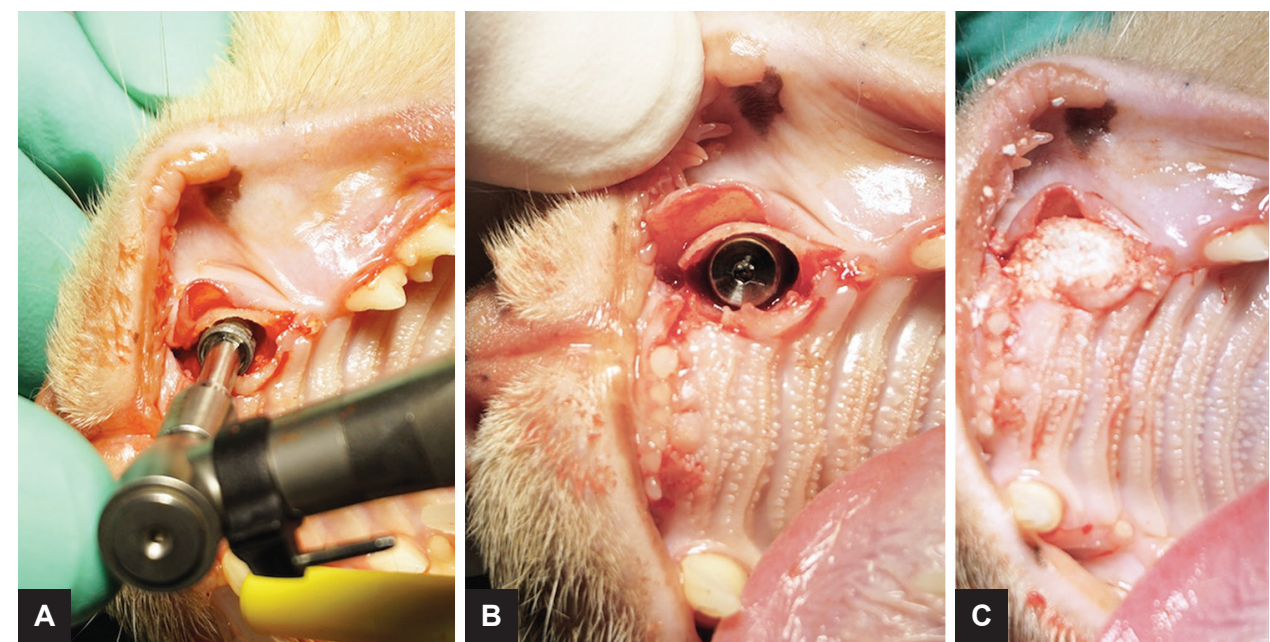

Figs 4A to C: (A) Surgical site during implant placement into the prepared osteotomy; (B) Void on mesial and distal following implant placement; (C) Placement of BA to fill the gaps and cover the implant prior to site closure 


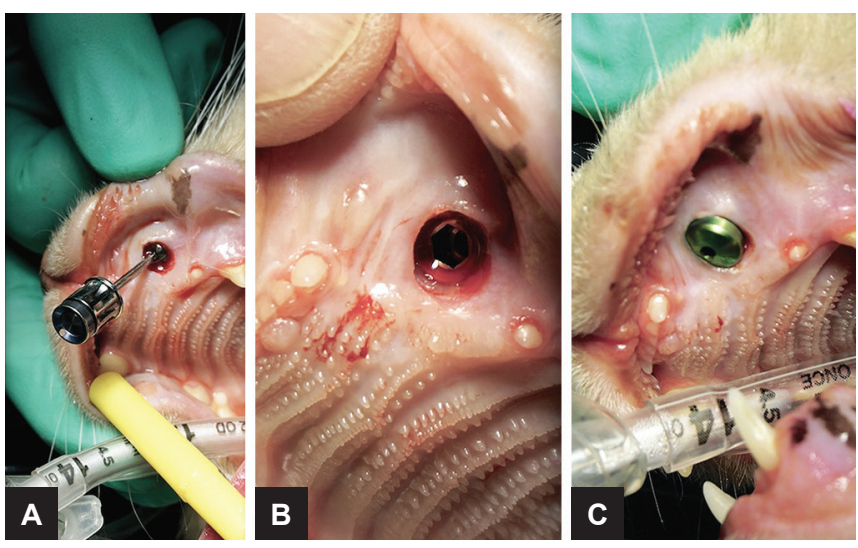

Figs 5A to C: (A) Reentry of the site at 6 months to begin restoration, implant (OCO Biomedical, Albuquerque, NM) was uncovered with a tissue punch (MWI Animal Health, Boise, ID) and cover screw (OCO Biomedical, Albuquerque NM) is being removed, (B) Implant without cover screw demonstrating healthy soft tissue; (C) Healing abutment placed into the uncovered implant

\section{Case 3}

A 6-year-old M/I Dachshund referred to evaluate and repair a traumatic fracture of the mandibular body.
Examination determined that the mandible had a vertical fracture extending through the superior and inferior cortex, leading to instability and malocclusion of the jaw. Radiographically, it was also noted that the molar (\#309), a mini root fragment, was imbedded in the distal mandibular fracture bone.

Following a full thickness flap of the fracture site, ridged fixation was attained with fracture reduction and a $\mathrm{T}$ plate (Henry Schein Animal Health, Dublin, $\mathrm{OH}$ ), spanning the fracture line, was placed to stabilize the site. Monocortical screws $(1.5 / 2.0 \mathrm{~mm}$ ) (Henry Schein Animal Health, Dublin, $\mathrm{OH}$ ) were inserted fixating the plate to the mandible. Bond apatite was packed into all voids and around the plate to completely cover the plate and fixation screws. Primary closure was achieved and no membrane was utilized and the site was closed with sutures. Bone apatite can be used to support internal fixation (plates and screws), bond loose bone and root fragments, fill voids, and act as a scaffold for rapid bone growth (Figs 7 to 12).
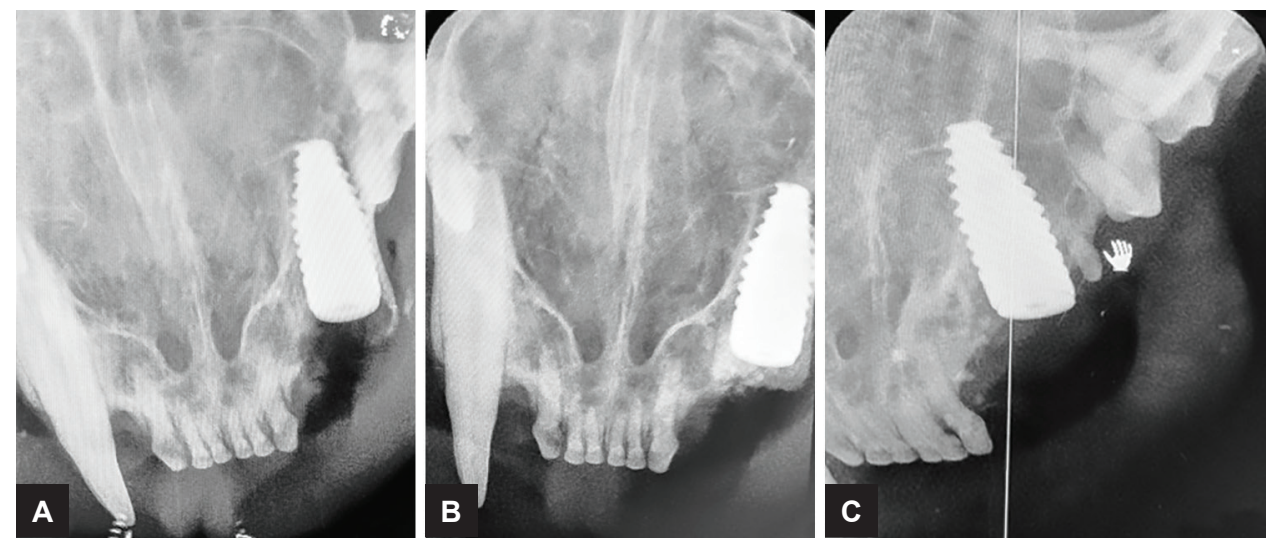

Figs $6 \mathrm{~A}$ to $\mathrm{C}$ : (Aand $\mathrm{B}$ ) Radiographs of the implant immediately following placement and site grafting ; (C) 6 months postimplant placement and grafting demonstrating maintenance of the bone around the implant

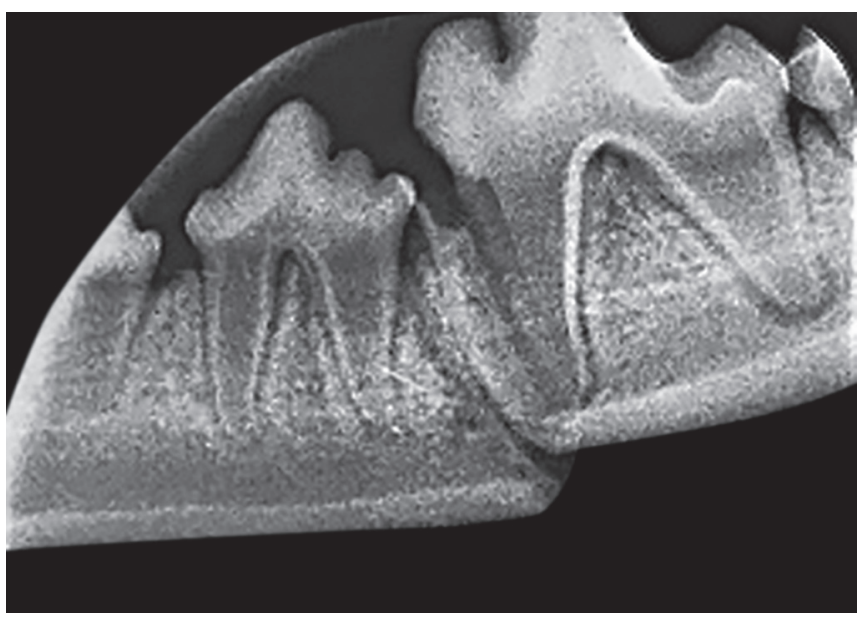

Fig. 7: Radiograph demonstrating the complete fracture of the mandible

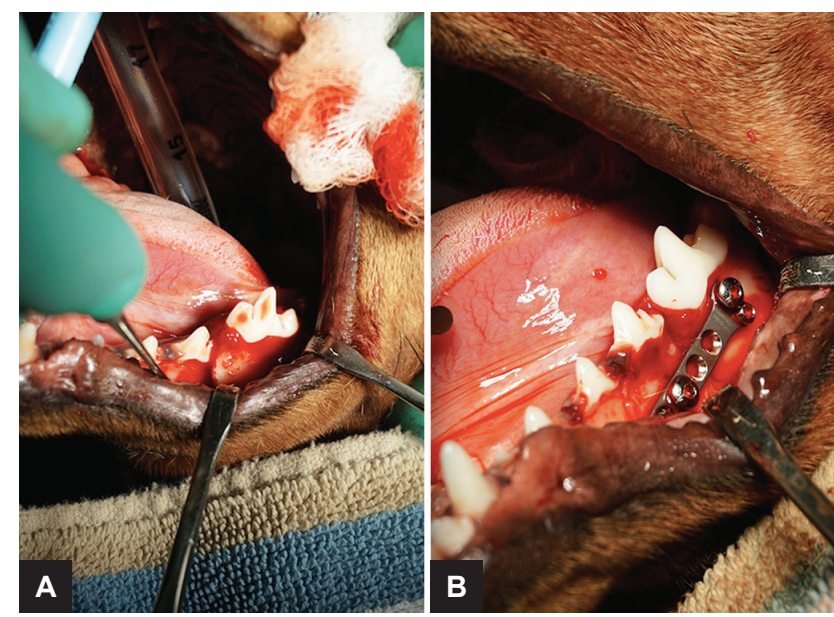

Figs $8 \mathrm{~A}$ and $\mathrm{B}$ : (A) Site following flap elevation demonstrating the mandibular fracture; (B) Reduction and stabilization with a mini T-plate secured with cortical self-tapping bone screws 


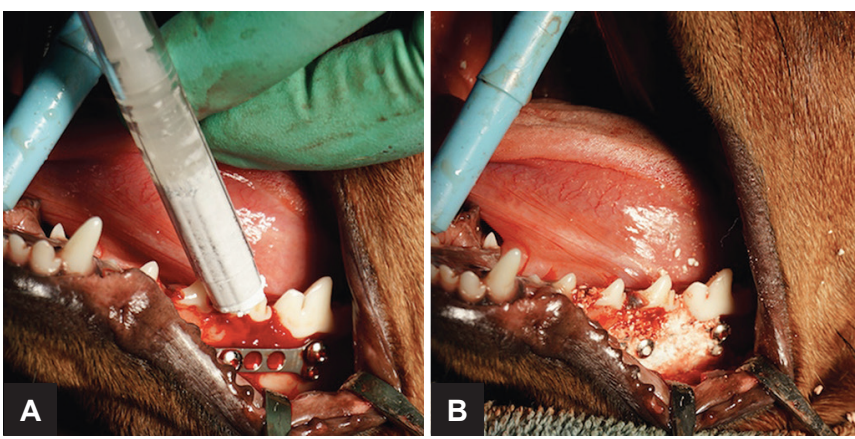

Figs 9A and B: (A) T-plate fixated to stabilize the fractured mandible and $B A$ being placed at the site; $(B)$ Fixation plate has been encased in BA prior to site closure

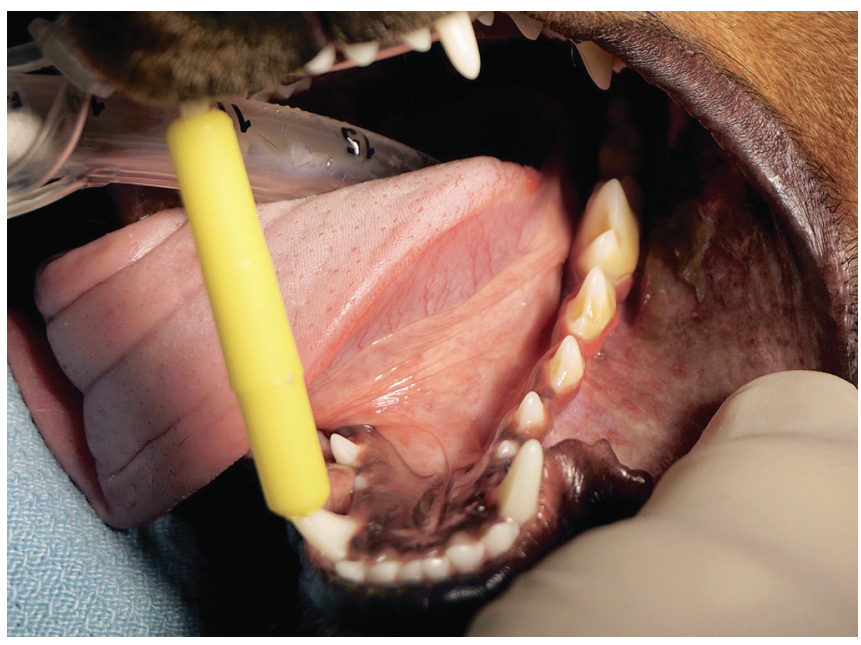

Fig. 11: Surgical site at 8 weeks postsurgery demonstrating healing with no inflammation and marginal stability of the soft tissue

\section{Case 4}

A 2-year-old M/N Siberian husky was treated for facial edema at the left suborbital area and referred to our clinic for a complete oral examination and dental X-rays under general anesthetic. Marked inflammatory tissue was noted with two draining fistulas apical to the attached gingiva in the alveolar mucosa at the left maxillary PM4 (\#208) site (Fig. 13). Dental radiographs revealed a generalized radiolucency associated with the roots of PM4, M1, and M (208, 209, and 210) (Fig. 14). Also present was generalized osteolytic sclerotic bone and an irregular trabecular pattern.

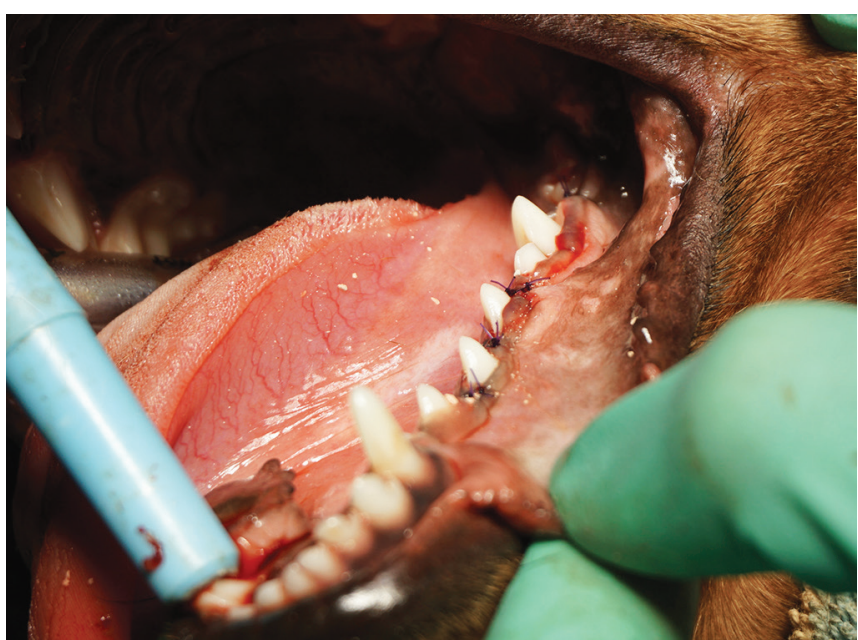

Fig. 10: Final primary closure with a tension-free flap

Treatment consisted of extraction of all affected teeth and radical surgical debridement of the associated area. Surgical debridement not only removes poorly vascularized infected bone, but also delivers well-vascularized tissue to the affected bone, facilitating the healing process and allowing antibiotics to reach the target areas. The surgical defect was filled with CS (BA) (Fig. 15). Tissue and bone samples were submitted for histopathological analysis which diagnosed osteomyelitis.

\section{RESULTS}

Surgical reentry at 9 weeks demonstrated vascularized bone in the grafted defect restoring ridge contours and no evidence visually of the CS placed at surgery (Fig. 15, right). Radiographs at 9 weeks demonstrated excellent graft and ridge maintenance and normal remodeling presentation of the CS with HA (BA) placed at surgery (Fig. 16). The surgical site at 6 months confirmed thick keratinized tissue and stability of the grafted ridge with restoration maintained to the ridge contours (Fig. 17).

\section{DISCUSSION}

Veterinary dentistry and oral surgery incorporate various biomaterials to augment extraction sockets and to rebuild
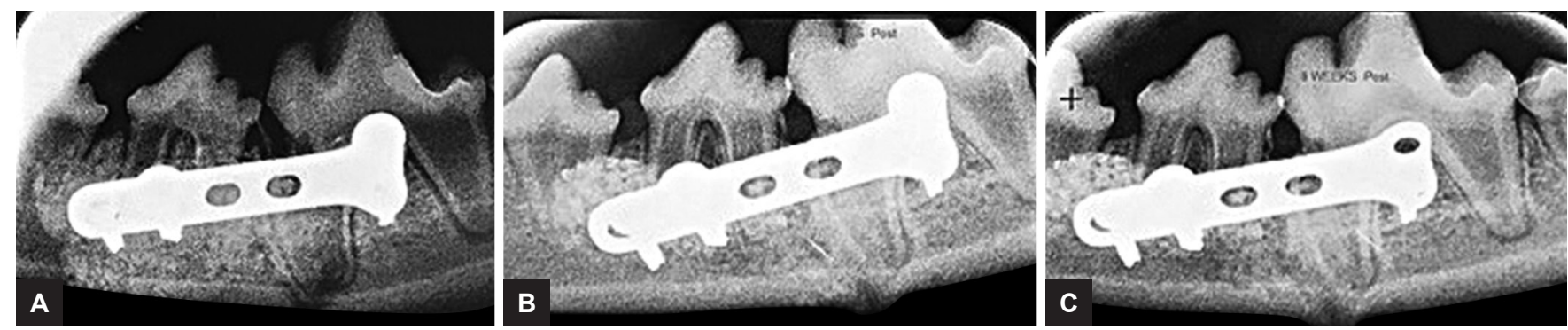

Figs 12A to C: (A) Radiograph of fracture reduction with mini plate; (B and C) At 8 weeks post-op demonstrating healed fractured site and new bone calcification 


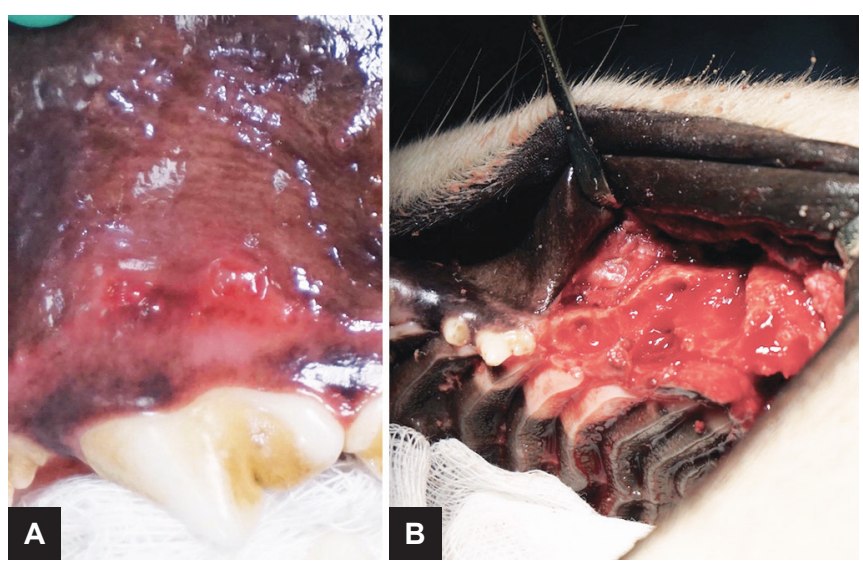

Figs 13A and B: (A) Draining fistulas at gingival and mucosal margin; (B) Extraction sites with radical debridement

extensive bony deficiencies. Current augmentation materials to enhance bone healing include: autologous grafts, xenografts, allografts, synthetics, blocks of bone, and putties. The cases presented demonstrate how bone cements are easy to use, minimally invasive, and require less surgical time to place while gaining complete functionality.

Bond apatite and 3-D bond are the next-generation CS bone grafting materials following the clinical track record of over 100 years of use as a graft material. The graft material is delivered with a self-contained selfmixing syringe injected directly into the surgical site. Bond Apatite is a slower resorbing material due to the addition of HA granule matrix and idea for larger bone defects that require longer space maintenance during the regeneration process.

The 3-D bond has complete resorption in 4 to 10 weeks and is recommended when smaller bone deficiencies, such as socket grafting with bony wall support are desired.

The clinical cases presented support the latest research data utilizing next-generation bone cements (BA and 3-D bond). Radiographic appearance studies demonstrate the maintenance of the alveolar ridge and reconstruction of the normal bony anatomy of larger defects. Additionally, the cements are biocompatible while supporting development of keratinized gingival tissue with minimal inflammation.

Surgical grafting procedures are simplified using this protocol with healing periods reduced to 12 weeks. Bone graft placement is reduced to place, press, and close with primary soft tissue closure. In most cases, no protective graft membrane is required. When primary closure is not achievable
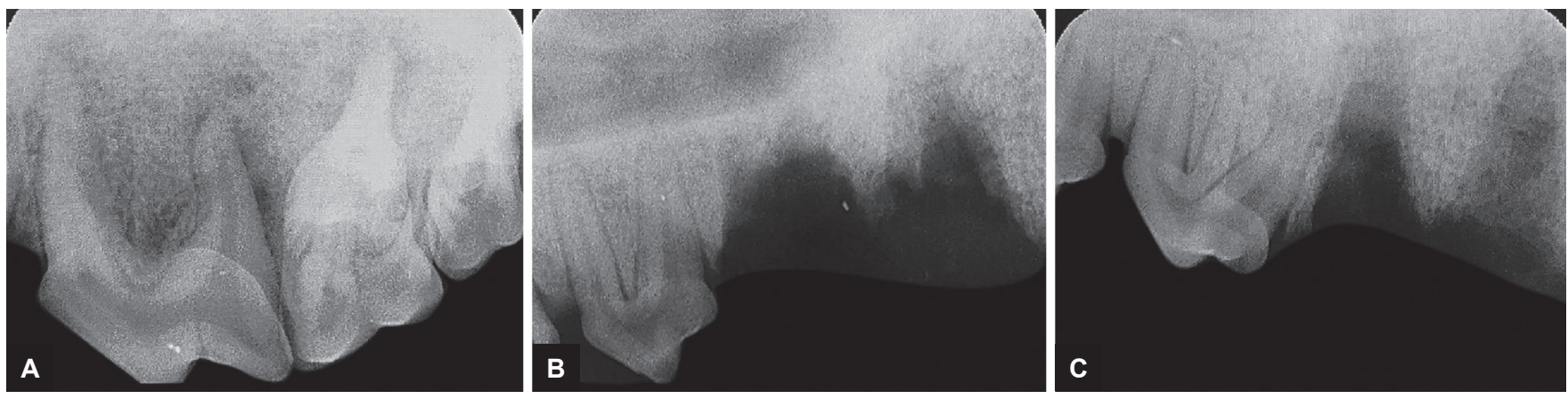

Figs 14A to C: (A) Initial radiographic presentation; (B and C) Radiographic presentation immediately following surgical extractions, and bone debridement

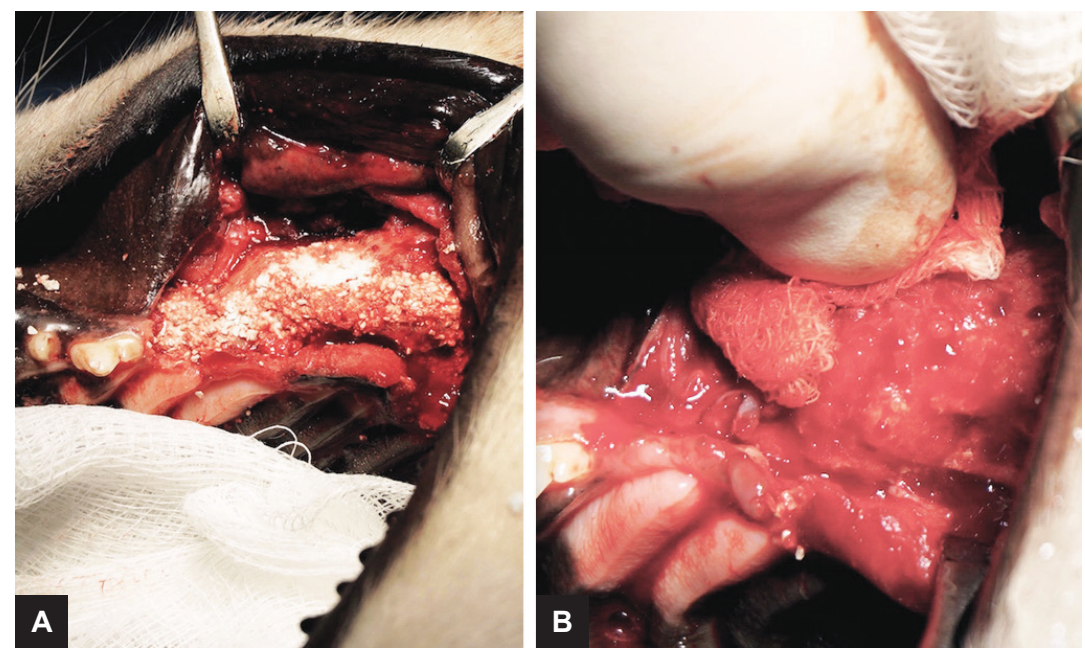

Figs 15A and B: (A) Bond apatite graft to support the maxillary defect; (B) Reentry at 9 weeks. Note the well-vascularized remodeling bone 

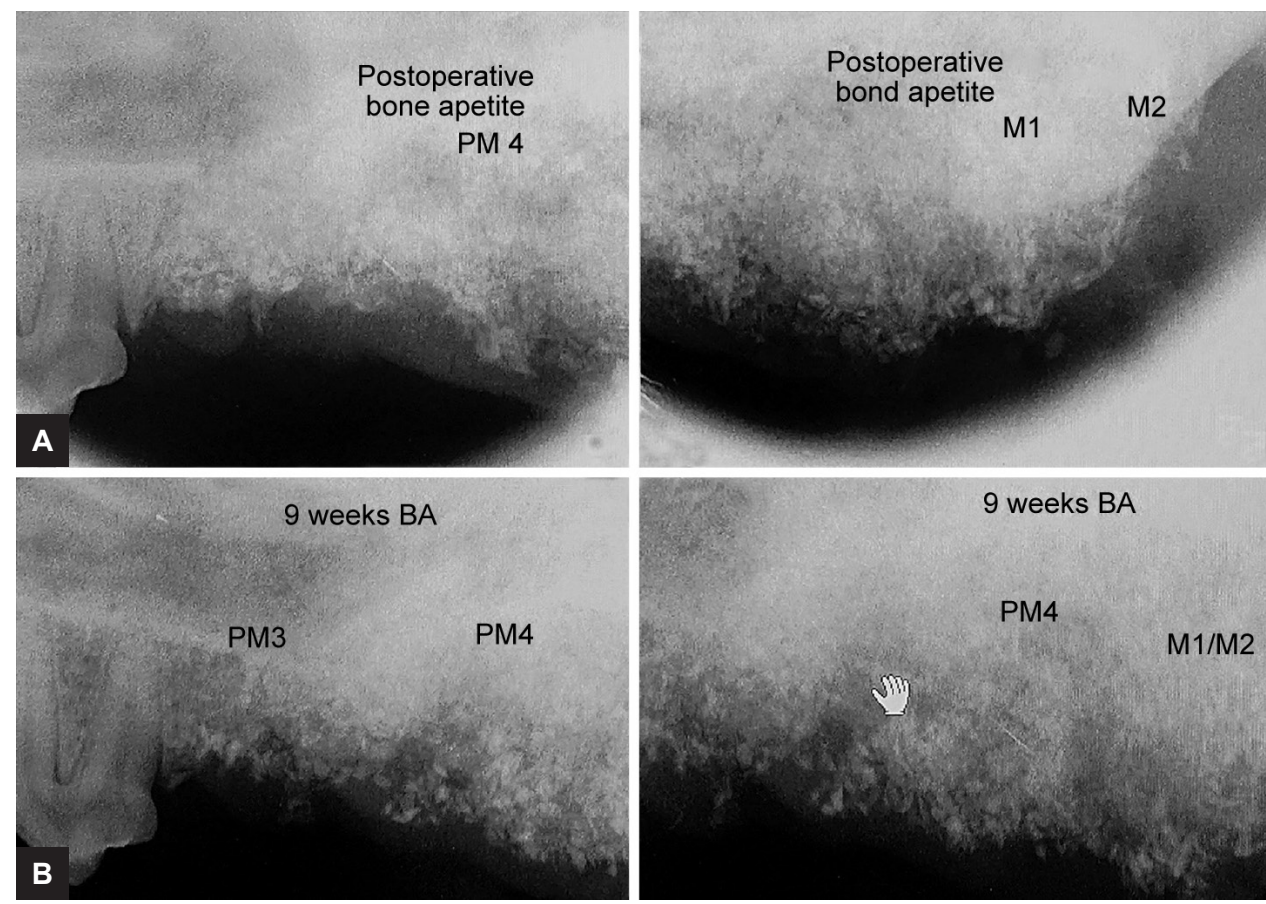

Figs 16A and B: (A) Immediately following BA graft placement; (B) Radiographs 9 weeks postoperative surgery

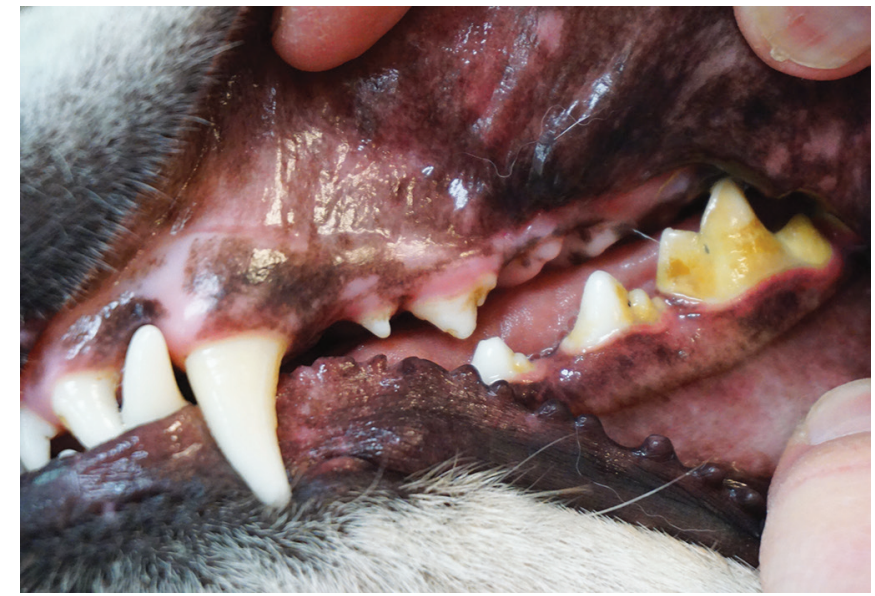

Fig. 17: Surgical site 6 months postsurgical demonstrating restoration of ridge contour with excellent soft tissue healing and minimal bone loss

and the gap is greater than 1 to $3 \mathrm{~mm}$, placement of a resorbable membrane is recommended to prevent volume loss during healing.

\section{CONCLUSION}

This case series demonstrates the clinical and biological advantage of the new biphasic CS bone graft substrate. Additionally, the product exhibits ease of use, adaptability to the site, radiographic bone integrity, and excellent support of keratinized tissue with no dimensional alterations. ${ }^{11}$

\section{REFERENCES}

1. Pietrzak WS, Ronk R. Calcium sulfate bone void filler: a review and a look ahead. The Journal of craniofacial surgery. 2000 Jul;11(4):327-333.

2. Bucholz RW. Non allograft osteoconductive bone graft substitutes. Clin Orthop Relat Res 2002 Feb;395:44-52.

3. Coetzee AS. Regeneration of bone in the presence of calcium sulphate. Arch Otolaryngol 1980 Jul;106(7):405-409.

4. Dreesmann H. Ueber knockenplombierung. Beitr Lin Chir 1892;9:804-810.

5. Peltier LF, Jones RH. Treatment of unicameral bone cysts by curettage and packing with plaster-of-paris pellets. J Bone Joint Surg Am 1978 Sep;60(6):820-822.

6. Peltier LF. The use of plaster of Paris to fill defects in bone. Clinical Orthopaedics and Related Research ${ }^{\circledR}$. 1961 Jan 1;21:1-31.

7. Peltier LF. The use of plaster of Paris to fill large defects in bone: a preliminary report. The American Journal of Surgery. 1959 Mar 1;97(3):311-315.

8. Peltier LF, Orn D. The effect of the addition of plaster-of-paris to autologous and homologous bone grafts in dogs. Surg Forum 1958;8:571-574.

9. Rosenblum SF, Frenkel S, Ricci JR, Alexander H. Diffusion of fibroblast growth factor from a plaster of Paris carrier. Journal of Applied Biomaterials. 1993 Mar;4(1):67-72.

10. Pecora G,AndreanaS, MargaroneJE3rd, CovaniU,ScottosantiJ. Bone regeneration with a calcium sulphate barrier. Oral Surg Oral Med Oral Pathol Radiol Endod 1997 Oct;84(4):424-429.

11. Horowitz RA, Rohrer MD, Prasad HS, Tovar N, Mazor Z. Enhancing extraction socket therapy with a biphasic calcium sulphate. Compend Contin Educ Dent 2012 Jun;33(6): 420-426. 